\title{
NUMERICAL EXPERIMENTS ON THE STABILITY OF SPHERICAL STELLAR SYSTEMS
}

\author{
M. HÉNON \\ Observatoire de Nice, 06300 Nice, France
}

The concentric shell model is used to investigate numerically the stability of spherical steady-state stellar systems. Polytropic models with an isotropic velocity distribution are found to be stable almost down to the limiting index $n=\frac{1}{2}$. 'Generalized polytropes', with a distribution function depending on energy and angular momentum, show instability when $n$ is low and the velocity distribution is radially elongated.

The full text of the paper has been published in Astronomy and Astrophysics 24, 229 (1973).

\section{DISCUSSION}

$P$. Bouvier: When you speak of a stable system, do you mean stable with respect to any kind of perturbations?

M. Hénon: No. The stability considered here is concerned only with perturbations preserving spherical symmetry. 